\title{
An Approach for Damage Identification and Optimal Sensor Placement in Structural Health Monitoring by Genetic Algorithm Technique
}

\author{
U. Muthuraman', M. M. Sai Hashita1, N. Sakthieswaran'1, P. Suresh², M. Raj Kumar33, \\ P. Sivashanmugam ${ }^{4}$ \\ ${ }^{1}$ Department of Civil Engineering, Anna University Regional Campus, Tirunelveli, India \\ ${ }^{2}$ Department of Electronics and Communication Engineering, National College of Engineering, \\ Maruthakulam, India \\ ${ }^{3}$ Department of Electrical and Electronics Engineering, National College of Engineering, \\ Maruthakulam, India \\ ${ }^{4}$ National Institute of Technology, Trichirapalli, India \\ Email: umram2410@gmail.com
}

Received 23 March 2016; accepted 13 May 2016; published 17 May 2016

Copyright (C) 2016 by authors and Scientific Research Publishing Inc.

This work is licensed under the Creative Commons Attribution International License (CC BY). http://creativecommons.org/licenses/by/4.0/

(c) (i) Open Access

\section{Abstract}

Civil engineering structures are constructed for strength, serviceability and durability. The structures thus constructed involve huge investment and labour work. In order to protect the structure from various damages, periodic monitoring of structures is necessary. Hence Structural Health Monitoring (SHM) plays a vital role in diagnosing the state of the structure at every moment during its life period. For this purpose, sensors are deployed in the structures for its efficient health monitoring. Sensors cannot be deployed at random locations of the structure. They have to be located at those points which reflect the damage. In this study, a 3-storey and a 4-storey building are taken and Modal Strain Energy (MSE) is used for finding the initial locations of sensors. The number of sensors obtained is then optimized using Genetic Algorithm (GA) technique. Finally damages are induced in certain locations of the structure and a damage detection technique called as "Flexibility Matrix Based Technique (FMBT)" is introduced for damage localization in the structure.

\section{Keywords}

SHM, Controlled Area Network, MLP-AGA, Sensor Placement 


\section{Introduction}

Structural Health Monitoring (SHM) is the process of comparing the current state of the structure with the original state persisted after construction. The state of the structure should always be within the safe limit as specified in the design. But this original state can be altered by aging of the structure, environmental conditions and by accidental loads acting on the structure [1]-[3]. The damage is usually characterized by change in the modal parameters of the structure [4]. Previously, frequencies and vibration based damage detection and several Non Destructive Techniques (NDT) were made use of in SHM. But the continuous monitoring of the structures was impossible by those NDT methods. Hence automated systems have to be developed by deploying sensors in certain locations of the structure to monitor its health from time to time. Various sensor placement techniques were introduced for determining the locations of sensors [5]. Generally for obtaining more information, more sensors have to be placed at the nodes of the structure. Placing a number of sensors is uneconomical and not possible in reality. Hence limiting the number of sensors comes into consideration. This process of limiting the sensors and placing them only at certain important nodes is termed as "Optimal Sensor Placement (OSP)" in structures [6].

Six different optimal sensor placements in buildings, namely Effective Independence (EFI), Optimal Driving Point (ODP), Non-Optimal Driving Point (NODP), Effective Independence Driving Point Residue (EFI-DPR), Singular Value Decomposition (SVD) and the Sensor Set Expansion (SSE) methods, are introduced by Pelin Gundes Bakir [7]. It was concluded that SSE technique was the best since it resulted in the homogenous distribution of sensors. Also an integrated methodology [8] has also been introduced by Pelin Gundes Bakir for efficient sensor placement and robustness of each technique is also presented and the Effective Independence method (EFI) was concluded as the best method. Kammer proposed a technique called "Effective Independence (EI) method" in which a number of candidate sensor positions were eliminated or added according to their ranks evaluated by the determinant of a Fisher Information Matrix (FIM) [9]. The relationship between the effective independence method and the modal kinetic energy method was presented by Li et al. [10] and a quick computation of the effective independence method through QR downdating was introduced. The most important stage is the selection of appropriate optimization algorithms for optimal sensor placement.

Carne and Dohrmann [11] proposed a famous algorithm called minMAC by distinguishing one modal vector from another to realize modal parameter identification. All of these algorithms have their own limitations so we go for traditional algorithm such as Simulated Annealing method [12], Particle Swarm Algorithm [13], Genetic Algorithm [14].

Among the above-mentioned heuristic algorithms, the use of Genetic Algorithm (GA) seemed to be an effective approach to sensor placement problems. Genetic Algorithms (GAs) are adaptive heuristic search algorithm based on the evolutionary ideas of natural selection and genetics. Genetic algorithms are a type of optimization algorithm, meaning they are used to find the optimal solution to a given computational problem that maximizes or minimizes a particular function. They imitate the biological processes of reproduction and natural selection to solve for the "fittest" solutions. The "traditional" GA is composed of a fitness function, a selection technique, and crossover and mutation operators which are governed by fixed probabilities [15]. The optimization problem using genetic algorithm is formulated by defining the sensor positions and the MAC as the design variables and the objective function, respectively. The fitness function is evaluated by the root mean square of the MAC matrix. The initial sensor placement is done by finding modal strain energy. One of the most important dynamic characteristics of the building is its mode shape. The modal parameters of the structure are obtained and the mode shape matrix is used to find the modal strain energy. Modal strain energy is a damage index which is proposed to detect multiple damages. The MAC matrix is obtained from the mode shape matrix [16]. This study focuses on the optimal sensor placement and damage detection of the structures. Section 2 proposes the methodology for optimal sensor placement. Section 3 presents the description of damage detection. Section 4 elucidates the analysis of results. Finally Section 5 concludes the paper.

\section{Methodology for OSP}

A combined optimization strategy called "MSE-GA" is opted for optimal sensor placement. For optimal sensor placement, the following methodology has been developed. Figure 1 depicts the methodology of MSE-GA method. 


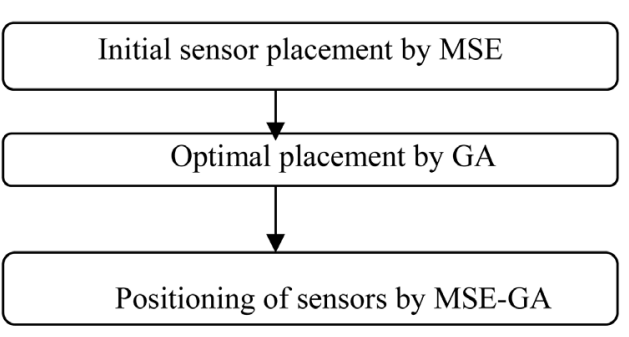

Figure 1. Steps of the MSE-GA method.

\subsection{Modal Strain Energy (MSE)}

The energy stored by a system undergoing deformation is called as strain energy. Modal Strain Energy technique is proposed as a damage index for initial sensor placement. MSE makes use of the modal parameters of the structure and hence prove to be an efficient means of detecting damage since the damage is generally reflected in the structure's modal properties. Out of "m" degree of freedom "n" locations that have high modal strain energy choosen for locating sensors. The MSE is obtained from the mode shape matrix and stiffness matrix it is given by,

$$
\text { MSE }=\phi^{\mathrm{T}} K \phi
$$

where, $\phi$ is the mode shape matrix and $K$ is the stiffness matrix. From the modal strain energy values the points of high modal strain energy is chosen for initial sensor placement in structures.

\subsection{Genetic Algorithm}

Genetic Algorithms are gaining wide attention by the research community. Genetic algorithm (GA) is rapidly growing area of Artificial Intelligence. It is categorised as subclass of evolutionary algorithms. It is applicable to large number of scientific problems. The various steps involved in GA are illustrated in Figure 2.

\subsubsection{Optimization}

Optimization is the process of making things better. Optimization can be defined as the science of determining the "best" solutions to mathematically defined problems. The process of optimization is shown in Figure 3.

The fundamental principle of optimization algorithm is "search for an optimal state". It can also be defined as the process of finding solutions that satisfy given constraints and achieve the objective at its optimal value.

\subsubsection{Flow Chart of GA}

\section{Fitness function}

The fitness function is the function that the algorithm is trying to optimize. The word "fitness" is taken from evolutionary theory. It quantifies how "fit" each potential solution is. The fitness function is one of the most pivotal parts of the algorithm. The fitness function is the only relation between the GA and the application itself, the function must be chosen with care. The fitness function must reflect the application appropriately with respect to the way the parameters are to be minimized. The fitness function is obtained from the MAC matrix and it constructed as,

$$
f=1-\text { RMS }
$$

where RMS is the root mean square of the off-diagonal elements in the MAC matrix.

\section{Modal Assurance Criterion (MAC)}

The Modal Assurance Criterion is defined as scalar constant which provides a useful criterion to evaluate the correlation of modal vectors. MAC matrix is defined as,

$$
\text { MAC }=\frac{\left(\Phi_{i}^{\mathrm{T}} \Phi_{j}\right)^{2}}{\left(\Phi_{i}^{\mathrm{T}} \Phi_{i}\right)\left(\Phi_{j}^{\mathrm{T}} \Phi_{j}\right)}
$$

where $\phi_{i}$ and $\phi_{j}$ are the $i$ th and $j$ th column vectors in the modal shape matrix $\phi$. The off-diagonal elements in the 


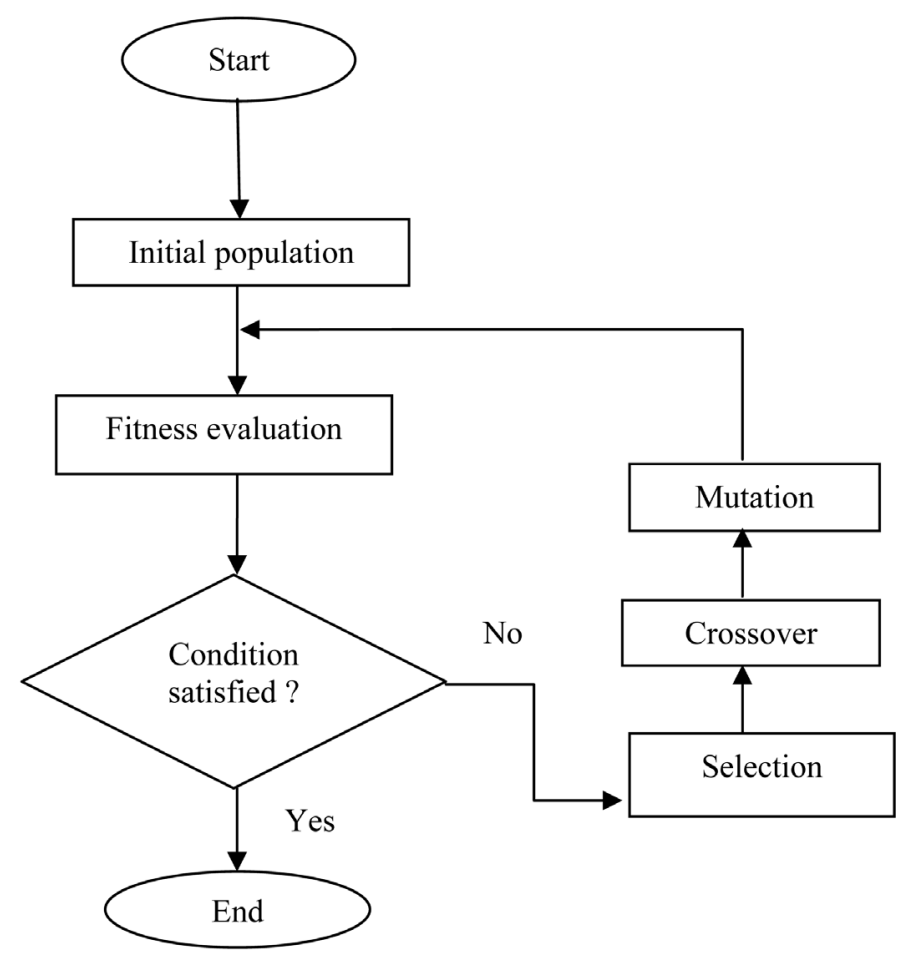

Figure 2. Flow chart of genetic algorithm.

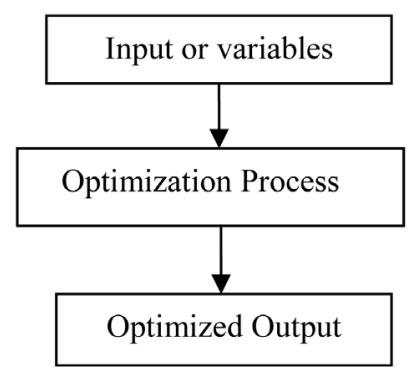

\section{Figure 3. Flow chart of optimization.}

matrix express the correlation between two modal vectors. The minimization of the off-diagonal terms in the MAC matrix gives a good MAC index that can guarantee the orthogonality of the measured modal vectors and increase the amount of modal information obtained from the collected data [16]. The possible range of the MACs is from zero to unity such that two modes $\phi_{i}$ and $\phi_{j}$ are the same when it is unity, while both are in no correlation when it is zero [17].

\subsection{Positioning the Sensor/Final Sensor Placement}

The sensor number which has the best fitness value is obtained from the genetic algorithm (GA) and it is designated as "Optimal sensor number" and these sensors are located at points of high modal strain energy (MSE) which obtained from initial sensor placement process. Thus, by using MSE-GA method optimal numbers of sensors are placed in the building which is referred to positioning of sensor or final sensor placement.

\section{Damage Detection}

For the purpose of damage detection, Flexibility Matrix Based Technique (FMBT) is introduced. It has been proved that the presence of damages increase the flexibility of the structure. The flexibility matrix $F$ is the inverse of the stiffness matrix $K$ relating the applied static forces $\{f\}$ to resulting structural displacements $\{u\}$ as 


$$
\{u\}=[F]\{f\}
$$

The relationship between the flexibility matrix and the dynamic properties of the structure is obtained by,

$$
\{f\}=\frac{1}{\omega^{2}} \Phi_{i} \Phi_{i}^{\mathrm{T}}
$$

where $\varphi_{i}$ is the mode shape and $\omega$ is the natural frequency of the structure [18].

\section{Change in Flexibility}

Damages are artificially introduced in the structure and the flexibility matrix for the damaged case is found. Now the change in flexibility is given by,

$$
\Delta f=\left\{f_{d}\right\}-\left\{f_{h}\right\}
$$

where $\left\{f_{d}\right\}$ is the flexibility matrix of the damaged case and $\left\{f_{h}\right\}$ is the flexibility matrix of the healthy structure.

\section{Result Analysis}

In this study, a 3-storey and a 4-storey building are taken and damage detection is done along with optimal sensor placement.

\subsection{3-Storey Building}

Modal analysis is carried out for the assumed 3-storey building with the mass matrix and stiffness matrix as inputs in Matlab. Figure 4 shows the modal strain energy plot of the assumed 3 storey structure. Figure 5 shows the fitness plot for all the nodes in the 3 storey structure. Figure 6 shows the final sensor placement after optimization through GA.

From Figure 4 it is clear that the energy is maximum at $2^{\text {nd }}$ and $3^{\text {rd }}$ storeys of the building. So 3 number of sensors is required in the initial sensor placement. In order to know that 3 number of sensors is the best solution, optimization is done. From Figure 5, it could be seen that maximum fitness is obtained at the sensor number 4 . Hence the optimal number of sensors is designated as 4 and this sensor is placed at the next higher energy storey of the building. Figure 7 shows the flexibility plot of the initial state. The damages are introduced at $1^{\text {st }}$ and $3^{\text {rd }}$ storeys of the building and the change in flexibility is noted. Figure 8 shows the change in flexibility of both damage cases 1 and 2 .

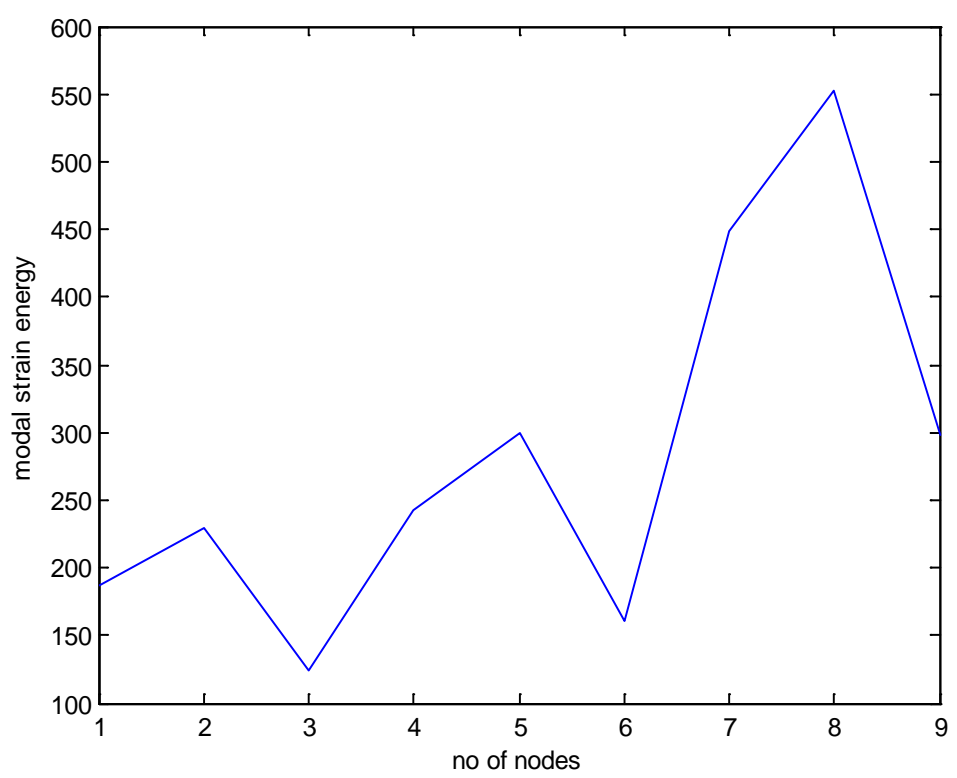

Figure 4. Modal strain energy plot of assumed 3-storey building. 


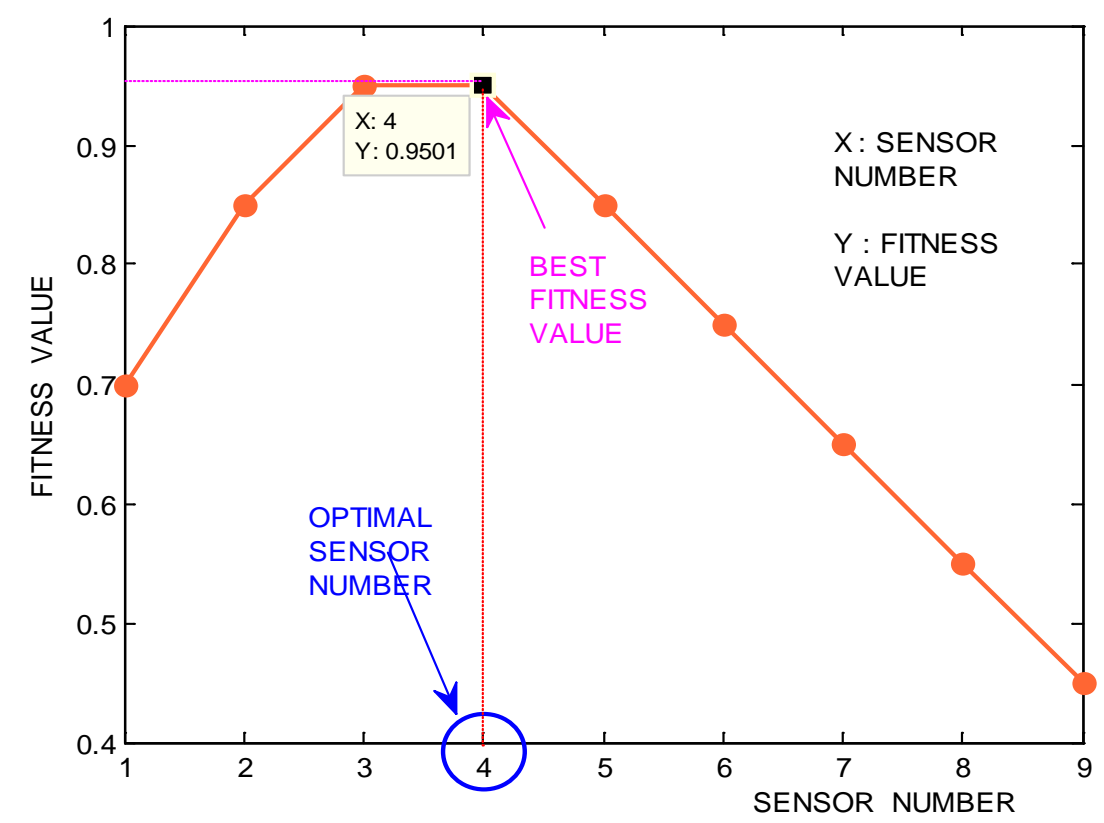

Figure 5. Fitness plot.

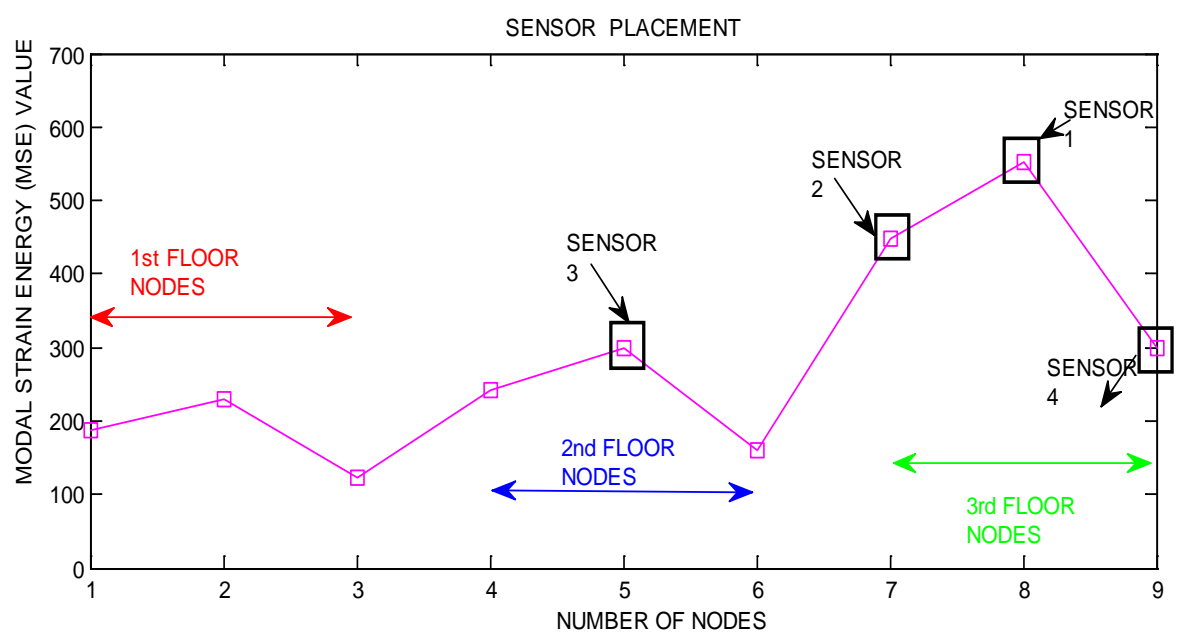

Figure 6. Positioning of sensors.

FLEXIBILITY WITHOUT DAMAGE

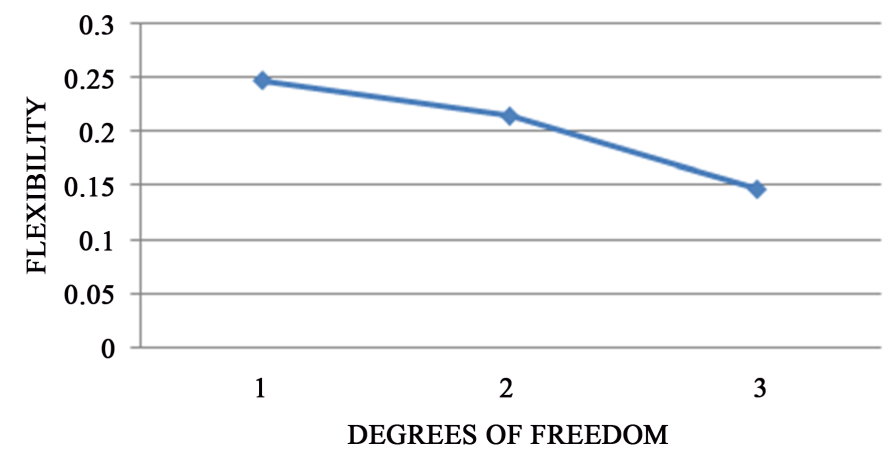

Figure 7. Flexibility plot for the undamaged case. 
From Figure 8, the severity of damage is understood and thus the damages are detected based on the change in flexibility. It could be noticed that by inducing the damages in any storey of the structure, its flexibility values change and it is possible to quantify the amount of damage in other storeys of the building.

\subsection{4-Storey Building}

Figure 9 shows the modal strain energy plot of the assumed 4-storey structure. From this figure, it is seen that 4 high energy points are present and hence 4 sensors are sufficient. Figure 10 shows the fitness plot for all the nodes in the 4-storey structure. This fitness plot shows that the highest fitness is obtained at sensor number 6 . Figure 11 shows the final sensor placement after optimization through GA and the optimized number of sensors are placed at high energy points of the building.

Figure 12 shows the flexibility plot of the initial state. The damages are introduced at $4^{\text {th }}$ and $2^{\text {nd }}$ storeys of the building and the change in flexibility is noted. Figure 13 and Figure 14 shows the change in flexibility of damage cases 1 and 2 respectively.

From Figure 13 and Figure 14, it could be seen that inducing damages in the $2^{\text {nd }}$ and $4^{\text {th }}$ storeys, has caused in changes in flexibility of other storeys as well and hence damage severity is known thereby.
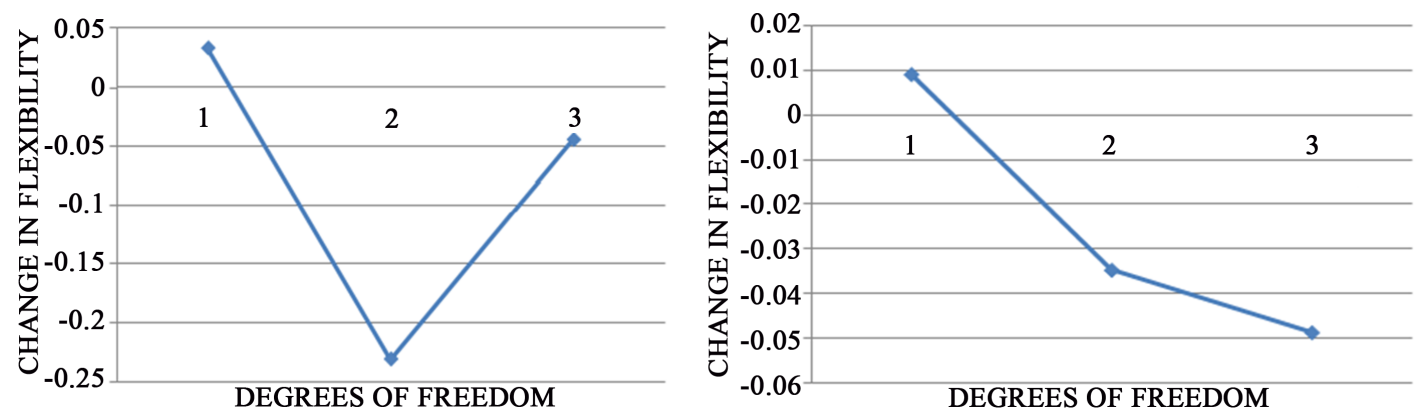

Figure 8. Plots showing change in flexibility.

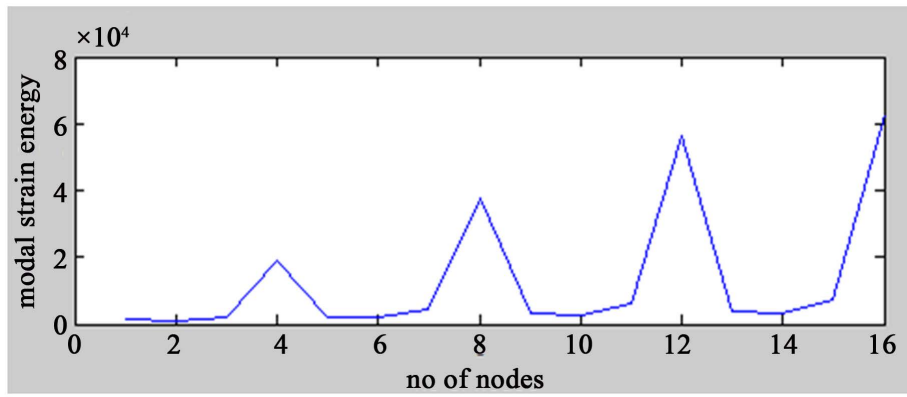

Figure 9. Modal strain energy of the assumed 4-storey.

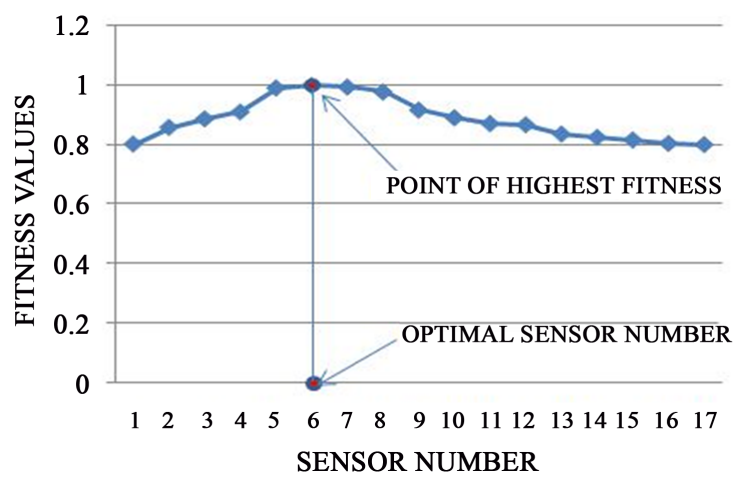

Figure 10. Fitness plot. 


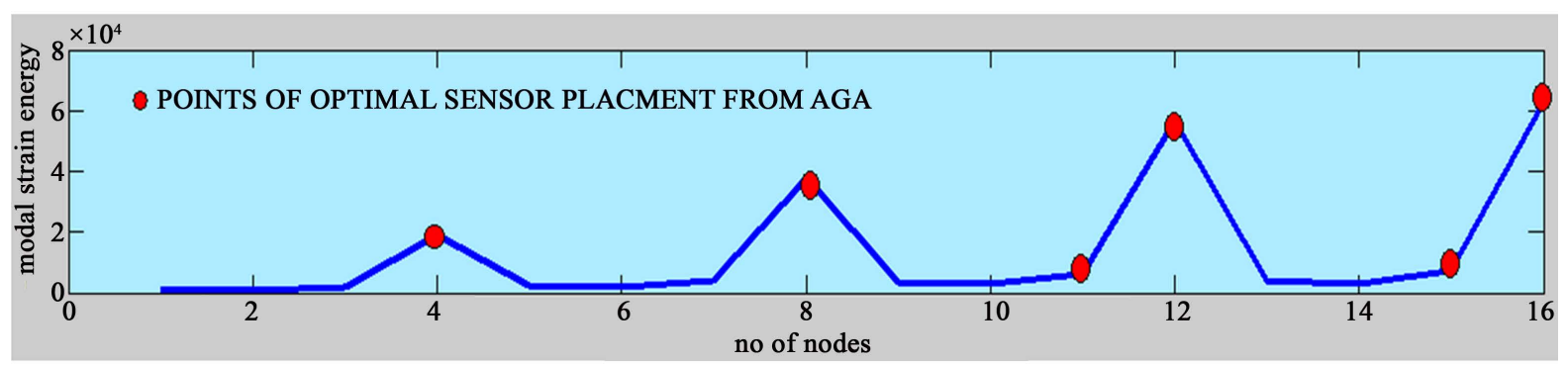

Figure 11. Final sensor placement.

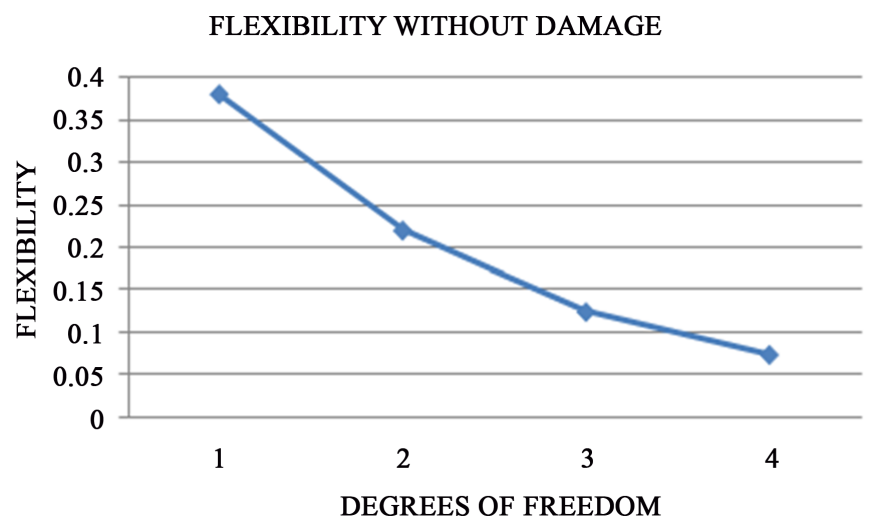

Figure 12. Flexibility plots for the initial state.

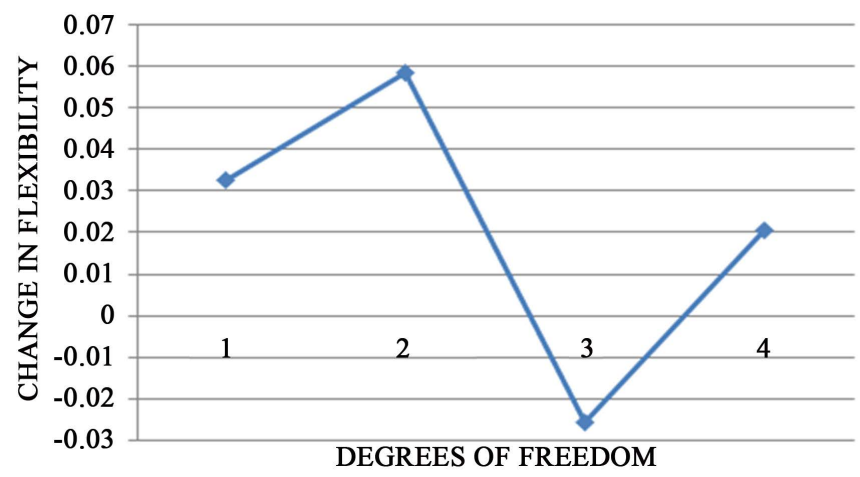

Figure 13. Plots showing change in flexibility in damage case 1.

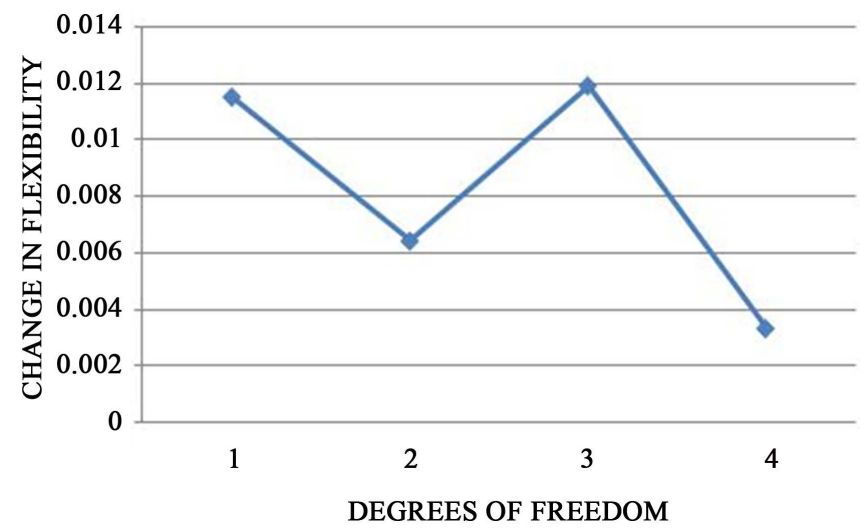

Figure 14. Plots showing change in flexibility in damage case 2. 


\section{Conclusion}

In this study a hybrid optimization and damage detection strategy "MSE-GA" and "FMBT" is introduced and hereby a 3-storey building and a 4-storey building are taken for the study. The initial number of sensors is found by MSE technique. The points of high MSE are chosen as points of initial sensors. These points are optimized using GA and the final sensors are placed at high MSE points. Finally damages are introduced in various storeys of the building and changes in flexibility are studied by "FMBT" technique. The conclusions made are summarized as follows: (i) The search space for the location of sensors is reduced by "MSE" technique. This concept of reducing the number of locations for the sensor placement superseded the older OSP techniques. (ii) The genetic algorithm technique is used for the optimization of the number of sensors obtained from initial sensor placement. This genetic algorithm makes use of the fitness function which is acquired from the root mean square of the MAC matrix. Thus the problem of efficient sensor placement in structures is made easy through the introduction of MSE-GA technique. (iii) FMBT technique is used for damage quantification and damage localization. Its performance is checked by inducing damages at various storeys of the building and change in flexibility for each storey is noted.

\section{References}

[1] Li, H.N., Yi, T.H., Yi, X.D. and Wang, G.X. (2007) Measurement and Analysis of Wind-Induced Response of Tall Building Based on GPS Technology. Advances in Structural Engineering, 10, 83-93. http://dx.doi.org/10.1260/136943307780150869

[2] Friswell, M.I. and Mottershead, J. (1995) Finite Element Modal Updating in Structural Dynamics. Kluwer Academic Publishers, Netherlands. http://dx.doi.org/10.1007/978-94-015-8508-8

[3] Ou, J. and Li, H. (2010) Structural Health Monitoring in Mainland China: Review and Future Trends. Structural Health Monitoring, 9, 219-231. http://dx.doi.org/10.1177/1475921710365269

[4] Boller, C., Chang, F.K. and Fujino, Y. (2009) Encyclopedia of Structural Health Monitoring. John Wiley \& Sons, New York. http://dx.doi.org/10.1002/9780470061626

[5] Yi, T.H. and Li, H.N. (2012) Methodology Developments in Sensor Placement for Health Monitoring of Civil Infrastructures. International Journal of Distributed Sensor Networks, 2012, Article ID: 612726.

[6] Worden, K. and Burrows, A.P. (2001) Optimal Sensor Placement for Fault Detection. Engineering Structures, 23, 885-901. http://dx.doi.org/10.1016/S0141-0296(00)00118-8

[7] Bakir, P.G. (2011) Evaluation of Optimal Sensor Placement Techniques for Parameter Identification in Buildings. Mathematical and Computational Applications, 16, 456-466.

[8] Bakir, P.G. (2008) An Integrated Methodology for Damage Identification in Existing Buildings Using Optimal Sensor Placement Techniques. The $14^{\text {th }}$ World Conference on Earthquake Engineering, Beijing, 12-17 October 2008, 1-11.

[9] Kammer, D.C. (1991) Sensor Placement for On-Orbit Modal Identification and Correlation of Large Space Structures. Journal of Guidance, Control, and Dynamics, 14, 251-259. http://dx.doi.org/10.2514/3.20635

[10] Li, D.-S., Li, H.-N. and Fritzen, C.-P. (2009) A Note on Fast Computation of Effective Independence through QR Downdating for Sensor Placement. Mechanical Systems and Signal Processing, 23, 1160-1168. http://dx.doi.org/10.1016/j.ymssp.2008.09.007

[11] Carne, T.G. and Dohrmann, C.R. (1995) A Modal Test Design Strategy for Model Correlation. Proceedings of the 13th International Modal Analysis Conference, New York, 13-16 February 1995, 927-933.

[12] Chiu, P.L. and Lin, F.Y.S. (2004) A Simulated Annealing Algorithm to Support the Sensor Placement for Target Location. Proceedings of the IEEE Canadian Conference on Electrical and Computer Engineering, Vol. 2, 867-870.

[13] Zhang, X., Li, J.L., Xing, J.C., Wang, P., Yang, Q.L., Wang, R.H. and He, C. (2014) Optimal Sensor Placement for Latticed Shell Structure Based on an Improved Particle Swarm Optimization Algorithm. Mathematical Problems in Engineering, 2014, Article ID: 743904.

[14] Gao, H. and Rose, J.L. (2006) Sensor Placement Optimization in Structural Health Monitoring Using Genetic and Evolutionary Algorithms. in Proceedings of SPIE Smart Structures and Materials, vol. 6174, San Diego,1687-1693.

[15] Srinivas, M. and Patnaik, L.M. (1994) Adaptive Probabilities of Crossover and Mutation in Genetic Algorithms. IEEE Transactions on Systems, Man and Cybernetics, 24, 656-667. http://dx.doi.org/10.1109/21.286385

[16] Brehm, M., Zabel, V. and Bucher, C. (2004) An Automatic Mode Pairing Strategy Using an Enhanced Modal Assurance Criterion Based on Modal Strain Energies. Journal of Sound and Vibration, 329, 5375-5392.

http://dx.doi.org/10.1016/j.jsv.2010.07.006 
[17] Yi, T.H., Li, H.N. and Wang, G.-X. (2008) Optimal Sensor Placement for Super High-Rise Building Based on Simplified Finite Element Model. Chinese Journal of Computational Mechanics, 25, 417-423.

[18] Montazer, M. and Seyedpoor, S.M. (2014) A New Flexibility Based Damage Index for Damage Detection of Truss Structures. Shock and Vibration, 2014, Article ID: 460692. 